\title{
La riabilitazione cardiologica: le Linee Guida e la realtà italiana
}

\author{
Pantaleo Giannuzzi
}

\begin{abstract}
Monaldi Arch Chest Dis 2006; 66: 121-128.
Dipartimento di Cardioangiologia Riabilitativa, Fondazione Salvatore Maugeri, IRCCS, Istituto Scientifico di Veruno.
\end{abstract}

\section{Introduzione}

Le malattie cardiovascolari sono la causa principale di morte in tutti i paesi del mondo occidentale, inclusa l'Europa. Inoltre, la malattia aterosclerotica, della quale la localizzazione coronarica è la più comune, è causa frequente di disabilità e di perdita di produttività, e contribuisce in modo sostanziale all'aumento progressivo dei costi sanitari soprattutto in rapporto all'invecchiamento della popolazione. Pertanto, nei pazienti con cardiopatia già documentata, la prevenzione di successivi eventi cardiovascolari, insieme al mantenimento di una adeguata capacità fisica ed autonomia funzionale ed una buona qualità della vita sono i principali obiettivi di salute pubblica. I risultati dell'EUROASPIRE II e di altri studi contemporanei indicano che troppi pazienti non ricevono appropriati interventi terapeutici o indicazioni sullo stile di vita e, di conseguenza, molti pazienti non raggiungono gli obiettivi raccomandati di prevenzione secondaria [1]. Pertanto, nonostante le raccomandazioni delle Associazioni Scientifiche sulla prevenzione delle malattie coronariche sia a livello locale-nazionale che europeo [2-12], l'implementazione delle strategie di prevenzione cardiovascolare nella pratica clinica è ancora largamente inadeguata.

Un intervento globale, multicomprensivo di riabilitazione cardiovascolare (RCV) è probabilmente l'approccio più efficace per una effettiva riduzione del rischio cardiovascolare e la cura a lungo termine di cardiopatici noti, così pure di soggetti ad alto rischio cardiovascolare a causa di un'elevata concentrazione di fattori di rischio coronarico [5-7, 11]. Le analisi economiche disponibili sull'argomento suggeriscono che la RCV è un intervento cost-effective dopo un evento coronarico acuto ed è economicamente giustificata. In particolare, la RCV attraverso programmi strutturati di prevenzione secondaria, è ora riconosciuta come componente essenziale di un moderno approccio e gestione del paziente con diverse manifestazioni della cardiopatia ischemica e con scompenso cardiaco e dovrebbe essere integrata in programmi di assistenza a lungo termine per tutti i pazienti con malattia cardiovascolare [13-25]. Ciò nonostante, sia la percentuale di pazienti proposti dai clinici per la riabilitazione che la disponibilità di programmi e servizi di RCV strutturati per una efficace prevenzione secondaria sono ancora largamente inadeguati sul territorio nazionale.

La RCV è ora riconosciuta il modello standard (standard of care) per il trattamento globale del pa- ziente cardiopatico in fase post-acuta o cronica e la forma più strutturata ed efficace di prevenzione secondaria, componente essenziale di un moderno programma assistenziale e di intervento a breve e lungo termine per tutti i cardiopatici, specie se con malattia coronarica e scompensati $[5,7,10,11,17,18,23-25]$.

\section{Evoluzione della riabilitazione cardiovascolare e definizione}

I primi programmi di RCV sono stati sviluppati negli anni '60 a seguito della documentazione dei benefici della mobilizzazione e deambulazione durante la ospedalizzazione prolungata dopo un evento coronarico. L'esercizio era la componente principale dei programmi iniziali che venivano essenzialmente proposti a pazienti sopravvissuti ad un infarto miocardico non complicato ed avviati, in genere, molto tardivamente dopo l'episodio acuto. Le preoccupazioni circa la sicurezza di programmi di esercizio fisico alla dimissione di un evento cardiovascolare hanno indotto lo sviluppo di programmi strutturati di riabilitazione, altamente controllati dai clinici e supervisionati con l'ausilio di monitoraggio elettrocardiografico. La sicurezza e i benefici di programmi supervisionati di training fisico di moderata intensità sono stati così largamente studiati e confermati $[7,11-14]$. Dati più recenti indicano che programmi non-supervisionati o domiciliari sono altrettanto efficaci e sicuri in pazienti ben selezionati e dopo opportune verifiche. Inoltre, gli effetti favorevoli dell'esercizio fisico sono stati ben dimostrati anche in pazienti con infarto miocardico esteso, con disfunzione ventricolare ed addirittura con scompenso cardiaco [11, 20-22, 25].

Sulla base delle evidenze scientifiche accomulate negli anni, l'Organizzazione Mondiale della Sanità (OMS) [3] ha definito la $R C V$ come un processo multifattoriale, attivo e dinamico che ha come fine ultimo quello di favorire la stabilità clinica, di ridurre le disabilità conseguenti alla malattia, di favorire un ruolo attivo nella società e, in ultima analisi, di ridurre il rischio di successivi eventi cardiovascolari, di migliorare la qualità della vita e di incidere quindi positivamente sulla sopravvivenza.

Pertanto gli obiettivi principali della RCV sono:

1) prevenire la disabilità conseguente alla malattia coronarica e cardiovascolare, particolarmente importante nei soggetti anziani e nei soggetti coinvolti in attività lavorative ad elevato impegno fisico;

2) prevenire successivi eventi cardiovascolari, ospedalizzazioni e morte cardiovascolare. 
Questi obiettivi si realizzano attraverso un approccio globale, integrato, di tipo clinico (diagnostico-valutativo ed assistenziale) e di intervento attivo, intensivo e a lungo termine per la ottimizzazione del profilo di rischio cardiovascolare globale.

Attualmente si riconosce che la combinazione di un adeguato approccio clinico, di esercizio fisico e di interventi educazionali e psico-comportamentali è la forma più efficace di RCV, sia per favorire la stabilità clinica e il recupero funzionale che per una adeguata prevenzione secondaria. Pertanto, la RCV non è solo indicata per pazienti disabili con limitazioni funzionali ma per tutti i pazienti con diagnosi di sindrome coronarica acuta e di infarto miocardico, per coloro che sono stati sottoposti a rivascolarizzazione coronarica mediante PTCA o by-pass chirurgico, pazienti con angina stabile, pazienti con claudicatio intermittens e pazienti con documentati fattori di rischio coronarico inclusa l'ipertensione, l'ipercolesterolemia e diabete. Inoltre, la RCV è indicata per pazienti con scompenso cardiaco cronico e per pazienti dopo trapianto di cuore [11].

Negli ultimi trenta anni, i modelli organizzativi di RCV hanno subito importanti variazioni in rapporto all'evoluzione demografica e alla caratteristiche dei pazienti, riflettendo largamente l'evoluzione ed i progressi nella cura globale del cardiopatico.

Nei primi anni della riabilitazione, la maggior parte dei pazienti arruolati in programmi di training fisico erano quelli che avevano subito un infarto miocardico non complicato. Negli anni successivi, anche pazienti post-infartuati con complicazioni venivano inclusi e considerati per una riabilitazione fisica sebbene di minore intensità e più graduale. La maggior parte dei pazienti che oggi vengono avviati a servizi e programmi di riabilitazione sono quelli che hanno subito un by-pass aorto-coronarico, una PTCA o altre forme di rivascolarizzazione miocardica. Con l'invecchiamento della popolazione, aumenta considerevolmente il numero di pazienti anziani avviati a RCV, molti dei quali hanno una coronaropatia severa e complicata, una diffusa patologia vascolare e gravi comorbidità $[4,5,12]$. Inoltre, molti pazienti una volta considerati a rischio troppo alto per programmi strutturati di riabilitazione, quali pazienti con ischemia miocardica residua, insufficienza ventricolare sinistra e scompenso cardiaco cronico, aritmie minacciose e stimolatori cardiaci (pace-maker, resincronizzazione ventricolare, ICD) traggono attualmente beneficio da programmi di training fisico supervisionato, più graduale e più protratto $[5,7,9,10,11,12,20-22,25]$. Ciò si realizza attraverso interventi integrati multifattoriali, in cui la componente fisica della riabilitazione si sviluppa in modo integrato e in combinazione con interventi per la stabilizzazione clinica e la ottimizzazione della terapia di prevenzione secondaria, con programmi di educazione alla salute, couselling, strategie comportamentali per un effettivo cambiamento dello stile di vita e interventi strutturati di tipo psicosociale per una effettiva riduzione del rischio coronarico e per il raggiungimento di altri obiettivi correlati sia allo stato di salute cardiovascolare che alla qualità della vita $[5,11,12,24]$.

Con il continuo ridursi della durata della ospedalizzazione per un evento coronario acuto, il de- condizionamento fisico conseguente è di solito minimo, ma il tempo speso in ospedale non è più adeguato per istruire i pazienti sulle cause e progressione della loro malattia, sulle modalità di monitoraggio dell'attività fisica, perché recepiscano adeguatamente il materiale educazionale e l'importanza di modificare lo stile di vita, e si adattino ed aderiscano a cambiamenti comportamentali necessari per la loro cardiopatia cronica. Pertanto, la possibilità di istruire $\mathrm{i}$ pazienti in merito alle possibilità di riduzione del rischio cardiovascolare, all'esercizio fisico ed ad un sano stile di vita è decisamente ridotta, in particolare dopo PTCA e per i pazienti più giovani non complicati, per i quali la lunghezza dell'ospedalizzazione è particolarmente breve.

In questo contesto, Linee Guida nazionali e autorevoli Agenzie di salute pubblica e di ricerca, hanno ampliato l'obiettivo dei programmi di RCV in rapporto alla valutazione e modificazione dei fattori di rischio, e hanno enfatizzato la grande necessità di programmi strutturati in ambito ospedaliero di riabilitazione degenziale e per pazienti ambulatoriali, oltre che di programmi di riabilitazione nella comunità e/o a domicilio, che siano strumento e riferimento per una efficace prevenzione secondaria nel territorio $[2,4,5,10-12,14,15]$.

\section{Le componenti principali dei Programmi e Servizi di Riabilitazione Cardiovascolare}

Da una estesa analisi della letteratura scientifica, che include autorevoli rapporti delle Associazioni Scientifiche Nazionali (Gruppo Italiano di Cardiologia Riabilitativa e Preventiva-GICR, ANMCO e SIC, Agenzia per i Servizi Regionali Sanitari), del Working Group on Cardiac Rehabilitation and Exercise Physiology della Società Europea di Cardiologia, delle Società Europee sulla prevenzione coronarica, dell'American College of Cardiology, dell'American College of Physicians, dell'American Heart Association e dell'American Association of Cardiovascular and Pulmonary Rehabilitation e una recente Cochrane analisi [2-5, 7-15, 22, 25] emerge che tutti i programmi e i servizi di RCV dovrebbero essere integrati in un processo multifattoriale a lungo termine che include: assistenza clinica e ottimizzazione della terapia o trattamento interventistico per rimuovere i sintomi; adeguata valutazione del rischio cardiovascolare; training fisico; educazione sanitaria e counseling riguardanti il rischio cardiovascolare e la riduzione del rischio; appropriati interventi comportamentali per un effettivo cambiamento dello stile di vita che coinvolgano i membri della famiglia per il raggiungimento di tali obiettivi; counselling professionale ed adeguato follow-up per assicurare compliance a lungo termine e motivazione per l'aderenza ai cambiamenti dello stile di vita e ai trattamenti farmacologici raccomandati.

I più immediati obiettivi dei programmi e servizi di RCV sono: perseguire la stabilità clinica, limitare gli effetti fisiologici e psicologici della malattia cardiovascolare, migliorare globalmente la capacità funzionale, e incidere così favorevolmente sul grado di autonomia, indipendenza e, quindi, sulla qualità della vita. Gli obiettivi a medio e lungo termine sono: ridurre il rischio di successivi eventi cardiova- 
scolari, ritardare la progressione del processo aterosclerotico e della cardiopatia sottostante ed il deterioramento clinico e, in buona sostanza, ridurre morbidità e mortalità. Pertanto, gli obiettivi di prevenzione secondaria assumono importanza prioritaria e sono inclusi negli obiettivi generali della RCV, che in buona sostanza può essere vista come l'applicazione clinica della prevenzione cardiovascolare per mezzo di un approccio professionale, integrato, multidisciplinare per la effettiva riduzione del rischio coronarico e la cura a lungo termine dei pazienti cardiopatici, così come dei soggetti ad alto rischio con elevata concentrazione di fattori di rischio cardiovascolare.

I benefici e gli effetti preventivi della RCV documentati in letteratura sono: riduzione e controllo dei sintomi, miglioramento della tolleranza allo sforzo e della capacità lavorativa, miglioramento dell'assetto lipidico, metabolico e del profilo di rischio cardiovascolare globale, riduzione del fumo, miglioramento della capacità di gestione dello stress e della vita di relazione e, in generale, maggiore sensazione di benessere. Tutto questo si associa ad attenuazione del processo aterosclerotico con significativa riduzione della incidenza di successivi eventi cardiovascolari, delle ospedalizzazioni e, in ultima analisi, di morbidità e mortalità totale.

A dispetto di questi evidenti benefici, meno di un terzo dei pazienti eleggibili per RCV di fatto partecipano a programmi formali di riabilitazione nella maggior parte dei paesi europei [11, 19, 23, 25]. Le barriere alla RCV sono da attribuire a: le scarse risorse, problematiche culturali, attitudinali e strutturali, maldistribuzione geografica dei servizi disponibili, eccessive distanze, carenza di strutture adeguate, insufficiente programmazione e finanziamenti inadeguati. Ulteriori barriere sono: l'incapacità dei medici a valutare le necessità e la motivazione dei pazienti ai cambiamenti dello stile di vita, insieme alla scarsa abilità a valutare i progressi degli interventi, nonché la scarsa attitudine dei medici (non sempre per ragioni culturali) ad avviare i pazienti, in particolare le persone più anziane e le donne, ai programmi di RCV di fatto disponibili. Questo è vero, a dispetto del fatto che i medici non hanno in generale le appropriate competenze o il tempo necessario per fornire ai pazienti efficaci interventi per una corretta alimentazione, la gestione del peso, l'interruzione del fumo, i programmi di attività fisica, l'aspetto psicosociale e comunque il counseling occupazionale. In realtà la RCV, in molte Università, non è ancora una componente del curriculum formativo medico, sia della medicina di base che della cardiologia.

La realizzazione di servizi di RCV in ambito ospedaliero o in Centri specializzati in grado di fornire un intervento globale e un approccio integrato multifattoriale attraverso operatori esperti dedicati (cardiologi, infermieri, fisioterapisti, fisiologi, psicologi, specialisti comportamentali e dietisti, tutti con adeguato training ed esperienza professionale specifica), e l'implementazione di programmi territoriali per la riduzione del rischio cardiovascolare a lungo termine, coordinati con i medici di base, costituiscono certamente le modalità più efficaci ed efficienti di RCV.

\section{Istanze emergenti per l'organizzazione e sviluppo della RCV}

Con il progressivo invecchiamento della popolazione generale, le caratteristiche demografiche e cliniche dei pazienti ospedalizzati per un evento cardiovascolare o sottoposti a cardiochirurgia stanno cambiando rapidamente. Questa popolazione è ora più anziana ed aumenta la prevalenza di donne sottoposte a cardiochirurgia anche di età avanzata; si tratta spesso di pazienti con malattia coronarica trivasale, estesa malattia vascolare, disfunzione ventricolare sinistra a decorso complicato. Inoltre, la prevalenza di gravi comorbidità quali diabete, malattie cerebrovascolari, sequele neurologiche, insufficienza renale e respiratoria e decadimento fisico tra i pazienti ricoverati è impressionante. Dato l'invecchiamento della popolazione, il numero di pazienti con scompenso cardiaco ed i costi del sistema sanitario stanno crescendo vertiginosamente. Tutti questi pazienti hanno assoluto bisogno di assistenza clinica, di interventi specifici, di supporto psicosociale dopo la fase acuta e di programmi strutturati di continuità asssistenziale.

Di rilievo, visto la pressante sollecitazione ad accorciare la durata del ricovero durante la fase acuta, non rimane tempo a sufficienza, da un lato, per una verifica della stabilità clinica e una accurata stratificazione prognostica e, dall'altro, per facilitare il recupero funzionale e l'acquisizione di conoscenze in merito alla gestione della malattia e all'acquisizione di un corretto stile di vita. Per queste ragioni, si intravede una forte necessità di programmi strutturati di RCV di tipo degenziale, soprattutto per i pazienti ad alto rischio e i non autosufficienti, per favorire la stabilità clinica, agevolare il ritorno ad una vita indipendente a domicilio e la aderenza ad un programma individualizzato di gestione della malattia a lungo termine per una efficace prevenzione secondaria.

Autorevoli e dettagliati documenti relativi alle strutture organizzative, alle modalità attuative, e all'approccio ai servizi di RCV sottolineano l'importanza della stratificazione del rischio, della valutazione dell'autonomia funzionale, dei bisogni assistenziali e sociali nei pazienti cardiopatici come base per interventi terapeutici e riabilitativi individualizzati, inclusi la prescrizione del training fisico con appropriata supervisione, l'intervento educazionale e comportamentale e l'impostazione del follow-up [2, 5, 11, 12].

La complessità e l'intensità di tale approccio saranno commisurate alle caratteristiche cliniche, ai bisogni assistenziali e al grado di autonomia dei pazienti. Pazienti complicati e ad alto rischio dovrebbero pertanto essere indirizzati a strutture riabilitative degenziali a più alto livello diagnostico ed organizzativo; pazienti invece a medio o basso rischio possono essere efficacemente gestiti presso strutture riabilitative di livello organizzativo intermedio o ambulatoriale. Particolare importanza sta assumendo la riabilitazione in pazienti anziani, specie dopo cardiochirurgia, e con scompenso cardiaco, nei quali il controllo delle complicanze, la stabilità clinica, la ottimizzazione del profilo di rischio e il mantenimento di un sufficiente grado di autonomia funzionale sono di estrema rilevanza clinica e sociale. 
Di fatto, differenti livelli di programmi riabilitativi sono erogati in ambito ospedaliero da team specializzati: RCV in regime di degenza (riabilitazione degenziale) per pazienti a medio-alto rischio, disabili e più complessi; RCV in regime ambulatoriale (riabilitazione ambulatoriale) per pazienti più indipendenti, a basso rischio e clinicamente stabili che richiedono una minore supervisione.

Sebbene gli obiettivi siano identici a quelli dei programmi di riabilitazione ambulatoriale, i programmi di riabilitazione degenziale sono specificatamente strutturati per fornire interventi più intensi e/o complessi, e hanno il vantaggio di essere in grado di iniziare precocemente dopo l'evento acuto, di includere pazienti più complessi, a medio-alto rischio o clinicamente instabili, di includere pazienti disabili e/o pazienti più anziani (in particolare quelli con co-morbidità) e, pertanto, di facilitare il passaggio dalla fase ospedaliera ad una condizione clinica più stabile ed una vita indipendente a domicilio. Uno dei maggiori svantaggi dei programmi residenziali è la relativa breve durata dell'intervento in merito alla gestione dei fattori di rischio e dei cambiamenti dello stile di vita. Pertanto i programmi di riabilitazione degenziale dovrebbero essere seguiti da un programma ambulatoriale di lunga durata per la riduzione del rischio e la prevenzione secondaria, con adeguato monitoraggio clinico e funzionale. Sono stati recentemente sviluppati anche programmi di riabilitazione domiciliare, diretti da medici e coordinati da infermieri e/o fisioterapisti, risultati efficaci per favorire la continuità assistenziale e prevenire il deterioramento clinico, e utili per espandere l'erogazione di servizi di prevenzione secondaria nel territorio.

Gli esperti di settore raccomandano fortemente che i programmi di RCV si realizzino attraverso un approccio integrato, multifattoriale e multidisciplinare orientato alla riduzione del rischio cardiovascolare globale. Si riconosce, inoltre, che l'esercizio è spesso il veicolo per facilitare altre componenti della riabilitazione, quelle soprattutto legate allo stile di vita, inclusa la maggiore sensazione di benessere, ma che programmi che consistono di solo esercizio fisico non sono da considerare RCV a tutti gli effetti $[5,10-12]$.

\section{Modelli organizzativi}

L'OMS classifica i livelli della Cardiologia Riabilitativa in tre categorie in base alla qualifica del personale, alle dotazioni strumentali, alla complessità e alla specializzazione dell'intervento:

a) livello base, che prevede cure ed interventi nell'ambito della comunità (attraverso scuole, palestre, club);

b) livello intermedio, che si sviluppa all'interno di un ospedale cittadino;

c) livello avanzato, in un Centro di Riabilitazione, dove sono disponibili servizi e prestazioni di alta specialità.

Il livello base dovrebbe essere riservato ai pazienti a basso rischio, soprattutto indirizzato ai pazienti cronici, stabili, finalizzato a mantenimento del più alto grado di indipendenza, a promuovere un effettivo cambiamento dello stile di vita per una effi- cace prevenzione secondaria, e alla riduzione del rischio di nuovi eventi cardiovascolari.

I livelli intermedio o avanzato dovrebbero essere riservati ai pazienti nella fase precoce della malattia e a pazienti a rischio medio o elevato.

In effetti, i modelli di RCV che sono stati sviluppati in ambito ospedaliero da teams specializzati e dedicati comprendono la forma di riabilitazione degenziale per pazienti più complicati, instabili a medio-alto rischio e disabili, e la riabilitazione ambulatoriale per pazienti più autonomi, più stabili, a basso rischio e che richiedono minore supervisione.

La riabilitazione degenziale è specificatamente strutturata per garantire interventi più intensivi e complessi, per accogliere pazienti più precocemente dopo un evento cardiovascolare, è particolarmente raccomandata a pazienti ad alto rischio, più complicati, disabili specie se anziani, ed ha l'obiettivo di facilitare la transizione dall'ospedale verso una condizione clinica più stabile che permetta il mantenimento di una vita indipendente e possibilmente attiva a domicilio. Un limite della riabilitazione degenziale è la durata relativamente breve dell'intervento sullo stile di vita e fattori di rischio. Pertanto, i programmi di RCV in regime degenziale dovrebbero essere seguiti da un programma ambulatoriale di lunga durata di riduzione del rischio e prevenzione secondaria, che includa un adeguato monitoraggio clinico e funzionale, e programmi territoriali a lungo termine.

Le componenti principali della riabilitazione ambulatoriale, orientata alla prevenzione secondaria sono: la valutazione basale del rischio; l'assistenza clinica e l'ottimizzazione della terapia finalizzate a rimuovere i sintomi ed ottenere una adeguata stabilità clinica; interventi specifici per il recupero funzionale e training fisico per il mantenimento di una buona capacità funzionale ed uno stile di vita attivo; il supporto educazionale e il "counselling" per una efficace riduzione del rischio cardiovascolare e l'effettivo cambiamento dello stile di vita (intervento sui lipidi, ipertensione, fumo, sovrappeso, alimentazione); l'uso di interventi comportamentali e un adeguato follow-up. Tali programmi dovranno essere sviluppati in un contesto ambulatoriale e condotti da personale specializzato e dedicato. La riabilitazione ambulatoriale è raccomandata per pazienti a basso rischio, e la durata di 8-12 settimane è considerata adeguata per sviluppare in modo appropriato tutte le componenti della riabilitazione ai fini di una efficace prevenzione secondaria.

A completamento del programma iniziale di riabilitazione e prevenzione secondaria, i pazienti dovrebbero essere orientati verso programmi più semplici di mantenimento a lungo termine nel territorio con il supporto di iniziative e servizi (clubs coronarici, telecardiologia) nella comunità (figura 1).

\section{Indicazioni per la riabilitazione degenziale}

In generale, la riabilitazione degenziale, sia nell'ambito di un ospedale cittadino o in un grande Centro di Riabilitazione, è preferibile per i pazienti a rischio intermedio o elevato, specialmente per quelli con decorso della fase acuta complicato o con persistente instabilità clinica, in modo da ottenere 


\section{Current directions for Cardiac Rehabilitation POST-ACUTE PHASE}

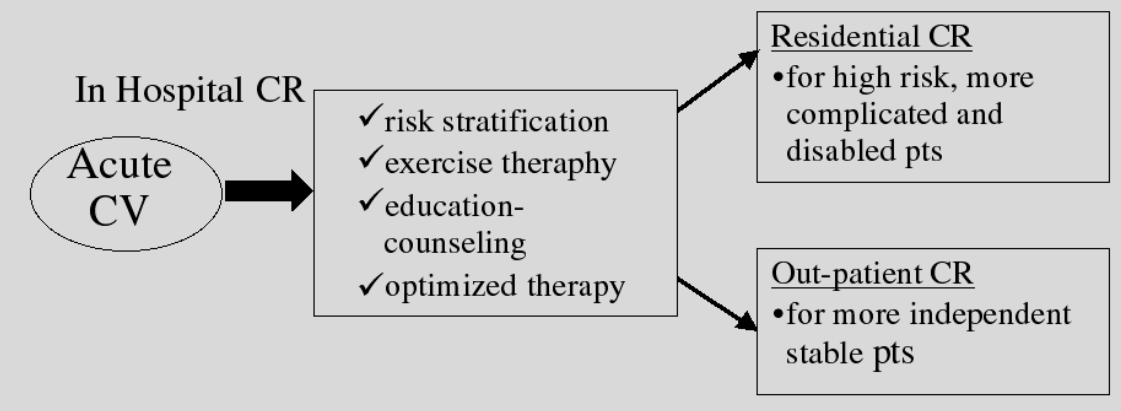

CHRONIC PHASE

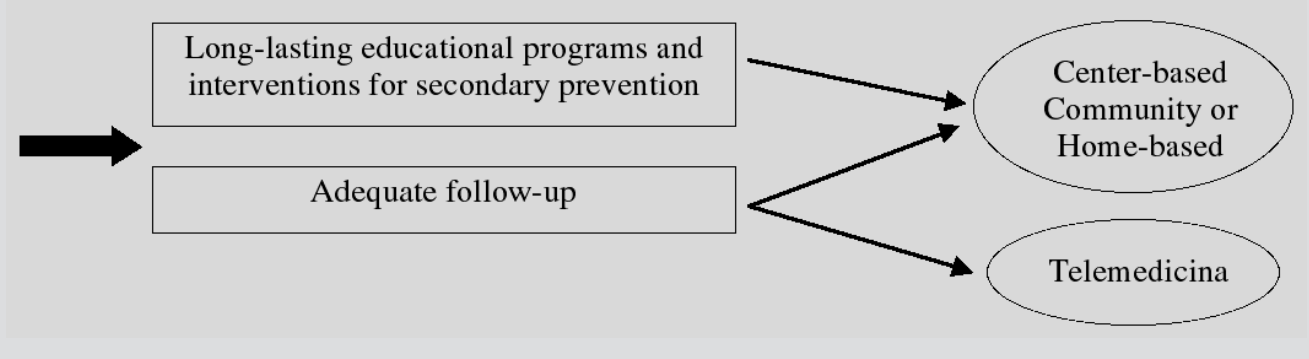

Figura 1.

condizioni cliniche più stabili e permettere un più rapido recupero funzionale.

I programmi di RCV sono riservati per le seguenti categorie di pazienti:

- pazienti con complicanze in fase acuta dopo infarto miocardio o angioplastica;

- pazienti dopo cardiochirurgia;

- pazienti con persistente instabilità clinica o complicazioni dopo l'evento acuto, o serie patologie concomitanti ad alto rischio di eventi cardiovascolari;

- pazienti con scompenso cardiaco avanzato (Classe III o IV), in particolare quelli candidati a trapianto cardiaco, e/o quelli che necessitano di terapie infusive continue o intermittenti e/o supporto meccanico;

- pazienti con recente trapianto cardiaco;

- pazienti dimessi precocemente dopo la fase acuta, anche non complicati, specie se sono anziani, di sesso femminile, o ad alto rischio di progressione della malattia coronarica, e

- pazienti non in grado di partecipare a programmi riabilitativi ambulatoriali per qualsiasi ragione logistica.

\section{Programmi e risorse in Riabilitazione degenziale}

La RCV degenziale di livello intermedio dovrebbe garantire assistenza clinica e la capacità di gestire ogni possibile emergenza; valutazioni non-invasive e valutazione funzionale per la stratificazione del rischio globale; training fisico supervisionato; programmi di educazione alla salute e raccomandazioni per cambiamenti dello stile di vita e riduzione del rischio; valutazione del profilo psicosociale e interventi comportamentali.
La RCV degenziale di livello avanzato (di alta specializzazzione) viene offerta in Centri altamente specializzati, dove sono disponibili servizi medici di elevato livello, personale altamente qualificato, multidisciplinarietà della cura, e assistenza continua. Questi Centri dovrebbero offrire tecniche diagnostiche noninvasive più complete e sofisticate (inclusa la cardiologia nucleare); procedure invasive (cateterismo cardiaco destro); valutazione ergometrica e occupazionale con appropriati interventi; accurato screening dei noti e meno noti fattori di rischio, compresa la determinazione dei fattori genetici soprattutto per i pazienti con coronaropatia giovanile; interventi educazionali e comportamentali e supporto psicosociale per popolazioni selezionate. Una unità di terapia sub-intensiva elo una unità per lo scompenso viene inoltre richiesta per il trattamento e il monitoraggio dei pazienti più complicati, clinicamente instabili, in particolare quelli con scompenso cardiaco avanzato e refrattario, quelli potenzialmente candidati o già in lista d'attesa per trapianto cardiaco e pazienti con gravi comorbidità e disabilità. A questi pazienti dovrebbe essere fornito un supporto clinico multispecialistico e interventi integrati, mirati alla stabilizzazione clinica ed alla autonomia funzionale che prevedano, attraverso forme di dimissione protetta e l'integrazione con il Medico di Medicina generale ed i servizi territoriali, il ritorno ad una vita domiciliare più indipendente, insieme a programmi strutturati di intervento e follow-up clinico in continuità assistenziale. Dopo il periodo degenziale, la $\mathrm{RCV}$ dovrebbe continuare ed essere integrata in un programma ambulatoriale di lunga durata in strutture di livello intermedio o avanzato, in base al livello di rischio, alla stabilità clinica, al grado di autonomia funzionale e disabilità. 


\section{Le Linee Guida Nazionali}

Le Linee Guida Nazionali dell'Agenzia dei Servizi Regionali e dell'Istituto Superiore della Sanità appena pubblicate [25], che hanno visto il coinvolgimento di tutte le società scientifiche (la Società Italiana di Cardiologia, l'ANMCO, la Federazione Italiana di Cardiologia, il GICR e anche la Federazione Italiana dei Medici di Medicina Generale), vogliono integrare il concetto di riabilitazione intesa come recupero della capacità funzionale globale con quello della prevenzione secondaria. Questo, ovviamente, è in linea con i documenti esistenti sia a livello europeo, che americano e soprattutto, in linea con le potenzialità della riabilitazione. In queste Linee Guida (rivolte essenzialmente ai medici di medicina generale, ai cardiologi, infermieri, fisioterapisti, fisiatri, psicologi, terapisti, e a tutti coloro che sono coinvolti nell'organizzazione del percorso) si sottolinea il fatto che troppo spesso i pazienti, dopo la riabilitazione, interrompono le abitudini acquisite per ciò che riguarda lo stile di vita, cioè non mantengono un'adeguata aderenza non solo, ai trattamenti farmacologici, ma anche alle misure di adeguato stile di vita, e questo è un altro aspetto che si vuole affrontare con dei modelli di integrazione ospedale-territorio. Si sottolineano ovviamente le potenzialità della riabilitazione in Italia, gli aspetti organizzativi, e, alla fine, ne è risultato un documento che può essere utile per la programmazione sanitaria e per la pianificazione degli interventi. Vengono sottolineati gli ostacoli alla riabilitazione, con l'obiettivo di rendere fruibile la riabilitazione cardiologica a tutti i cardiopatici, e si evidenzia soprattutto la discrepanza tra l'esiguo numero di pazienti che nell'attuale contesto accede ai programmi di riabilitazione ed i bisogni sanitari. Basta ricordare il bisogno più strettamente legato all'età. Si è avuto negli ultimi venti anni un incremento importante della durata della vita, con una percentuale di soggetti con più di 65 anni che è passata dal $13 \%$ al $19 \%$, con un incremento di oltre il $40 \%$. Il contesto sanitario è completamente cambiato: vi è un largo utilizzo di risorse, soprattutto di risorse ad alta tecnologia. Basta considerare il numero di angioplastiche ed interventi per via percutanea in 5 anni (dati del 2003 relativi al periodo 1998-2003 pubblicati sul sito del Ministero della Salute), con un aumento del $118 \%$ di angioplastiche, del 59-60\% di interventi di valvole, di circa il 40\% di impianti di pacemaker di tutti i tipi di device, di ricoveri DRG per insufficienza cardiaca, che sono aumentati in 5 anni del $16 \%$, con un numero di ricoveri che si avvicina ai 190.000 per anno. Il contesto di riabilitazione risente di questi importanti cambiamenti demografici della popolazione generale. In effetti, in un ambito di riabilitazione degenziale come quello della Fondazione Maugeri, la popolazione di cardiopatici che vi accedono è in rapida evoluzione verso caratteristiche di particolare complessità e disabilità. I DRG 144 (cardiopatia ischemica complicata) e 127 (scompenso cardiaco) rappresentano ormai oltre il 50\% di tutta la casistica di cardiopatici di Fondazione Maugeri. Inoltre, l'età media della popolazione è passata, in 5 anni, da 62 anni dell'anno 2000 a 67 anni del 2004, così pure la percentuale di grandi anziani di età $>75$ anni è aumentata progressivamente dal $13 \%$ dell'anno 2000 al $20 \%$ del 2004, e al 33\% nel sesso femminile.

Di fatto la popolazione che ora accede alle nostre strutture per riabilitazione degenziale, oltre che più anziana e tendenzialmente più fragile, ha maggiore frequenza di complicazioni e comorbidità ed è quindi gravata da più marcata disabilità dopo un evento cardiovascolare.

\section{Dall'Eccellenza all'Alta Specializzazione}

Nell'insieme aumenta rapidamente la popolazione di pazienti critici o complessi che richiedono un intervento sempre più articolato, multispecialistico ed intensivo dopo un evento cardiovascolare. Il cardiopatico complesso è insieme un paziente ad alto rischio cardiovascolare che di disabilità per la presenza di età avanzata, di elevata comorbidità, di disfunzione ventricolare sinistra severa, insufficienza cardiaca, e contemporanea compromissione dell'autonomia funzionale. La definizione di cardiopatico complesso in riabilitazione è riportata in tabella 1.

\section{Tabella 1. - Definizione del "cardiopatico complesso"}

A. Pazienti affetti da scompenso cardiaco cronico con necessità di terapie infusive (diuretici, inotropi, vasodilatatori) per recente instabilizzazione dopo un evento cardiovascolare o secondaria a progressione della patologia di base o a fattori instabilizzanti.

B. Presenza di aritmie ventricolari pericolose per la vita, sopraventricolari o bradi-aritmie mal tollerate emodinamicamente.

C. Cardiopatico sopravvissuto ad arresto cardiaco.

D. Cardiopatico con recente sindrome coronarica acuta nel quale l'efficacia della terapia farmacologica non sia stata ancora completamente valutata e/o ottimizzata.

E. Cardiopatici con ridotta autonomia e necessità di adeguati trattamenti farmacologici e lunghi interventi riabilitativi individuali a seguito di complicanze e/o comorbilità come:

- decadimento fisico e/o deficit cognitivi; specie se in soggetti di età avanzata) $\longrightarrow$ strumenti;

- scompenso cardiaco in atto;

- cospicuo versamento pleurico e/o pericardico;

- anemia significativa $(\mathrm{Hb}<8 \mathrm{mg} / \mathrm{dl})$;

- complicanze broncopolmonari significative (insufficienza respiratoria con EGA basale: $\mathrm{PaO} 2<50 \mathrm{~mm} / \mathrm{Hg}$, $\mathrm{PaCO} 2$ > 70-75 mmHg, $\mathrm{pH}<7.35$ );

- assistenza ventilatoria non invasiva;

- tracheostomia;

- complicanze neurologiche attive o croniche, con ridotta autonomia funzionale e necessità di assistenza nelle normali attività della vita quotidiana;

- insufficienza renale (filtrato glomerulare < 30\%);

- complicanze infettivologiche sistemiche;

- cattiva evoluzione delle ferite chirurgiche e/o presenza di decubiti;

- necessità di supporto nutrizionale (enterale e parenterale);

- lesioni periferiche su base vascolare e/o metabolica.

F. Pazienti in work-up o già in lista per trapianto.

G. Pazienti trapiantati. 
Si avverte quindi sempre più pressante la necessità di rispondere in modo più adeguato ed appropriato ai bisogni assistenziali ed al grado di disabilità/comorbidità del paziente cardiopatico che accede ai programmi di riabilitazione degenziale nell'attuale contesto sanitario. Proprio su questo punto, le recenti Linee Guida Nazionali [25] indicano un'importante raccomandazione clinico-organizzativa: “... per i pazienti clinicamente più complicati o instabili, si dovrebbero sviluppare programmi riabilitativi di alta specializzazione e complessità, che comprendano servizi medici di elevato livello, con possibilità di trattare adeguatamente, con un approccio multidisciplinare alla cura e assistenza continua, anche pazienti a rischio molto elevato, con disabilità e comorbidità".

In altre parole, esistono le premesse per evidenziare o per organizzare l'area di alta specializzazione in riabilitazione cardiovascolare riservata al paziente cardiopatico complesso.

L'organizzazione ed istituzione di un'area di alta specializzazione in riabilitazione cardiovascolare dedicata al cardiopatico complesso rappresenta una risposta potenzialmente di grande impatto in termini di salute pubblica se si vogliono affrontare in modo organico ed efficace le problematiche assistenziali, la complessità dell'intervento riabilitativo e quindi la gestione della fase post-acuta e cronica in continuità assistenziale di pazienti critici ad elevato rischio cardiovascolare e di disabilità.

L'Alta specializzazione in riabilitazione cardiovascolare è inteso come un modello integrato multispecialistico di riabilitazione ad elevato contenuto assistenziale, intensità e complessità riabilitativa per il cardiopatico complesso, che si rivolge essenzialmente a:

- cardiopatici più complicati, clinicamente instabili, a rischio elevato;

- pazienti con scompenso cardiaco avanzato e potenziali candidati al trapianto o già in lista d'attesa per trapianto;

- cardiopatici con grave comorbidità e disabilità che richiedono intervento complesso.

Le condizioni organizzative di carattere generale indispensabili per la realizzazione di una RCV di alta specializzazione sono:

- presenza di servizi medici di elevata professionalità;

- possibilità di gestire anche pazienti complessi e a rischio elevato;

- personale altamente qualificato;

- multidisciplinarietà della cura;

- assistenza continua.

Più in dettaglio, i requisiti generali per l'alta specializzazione sono:

a) disponibilità di una area critica di terapia intensiva/subintensiva con posti letto monitorati per i pazienti complicati e ad alto rischio e adeguato rapporto tra assistenza infermieristica e posti letto;

b) possibilità di eseguire tecniche diagnostiche non-invasive complete (inclusa la cardiologia nucleare o imaging vascolare non invasivo avanzato); procedure invasive (caterismo cardiaco destro); valutazione funzionale completa con appropiati interventi multispecialistici finalizzati al massimo grado di recupero e di autonomia funzionale; c) riconoscimento del Centro come RCV il riferimento (hub) per un bacino di utenza regionale o almeno sovra-provinciale;

d) riferimento per la gestione dei pazienti con scompenso cardiaco severo (come unità di terapia subintensiva e/o una unità per lo scompenso).

Requisiti specifici per l'alta specializzazione sono:

- monitoraggio dei pazienti al letto e/o in telemetria;

- possibilità di eseguire infusioni farmacologiche tramite pompe volumetriche;

- ultrafiltrazione;

- ecocardiografia ed emodinamica destra possibilmente in reparto;

- test cardiopolmonare;

- ECG dinamico;

- studio del sistema nervoso autonomo;

- studio dei segnali cardiorespiratori a riposo e in esercizio;

- valutazione e trattamento dei disturbi respiratori nel sonno;

- valutazione metabolico-nutrizionale;

- interventi clinici integrati multispecialistici a supporto del cardiologo responsabile del progetto di cura (neurologo, fisiatra, pneumologo, internista, infettivologo, chirurgo, cardiochirurgo, nutrizionista...); fisico;

- intervento integrato di fisioterapia e training

- valutazione psicologica e psichiatrica;

- supporto psicologico e psicosociale;

- programmi educazionali;

- continuità assistenziale.

Crediamo che la programmazione ed istituzione di RCV di alta specializzazione in Centri di riabilitazione multispecialistaici con un bacino di utenza sovraprovinciale o regionale (dove sono disponibili servizi medici di elevato livello, con possibilità di gestire anche pazienti complessi e a rischio molto elevato, personale altamente qualificato, multidisciplinarità della cura, e assistenza continua) rappresenti una adeguata risposta ai crescenti bisogni di molti cardiopatici nella fase post-acuta e cronica, con l'obiettivo di favorire la stabilità clinica, l'autonomia funzionale, la continuità assistenziale per prevenire le riospedalizzazioni ed una vita indipendente a domicilio.

\section{Bibliografia}

1. EUROASPIRE II Study Group. EUROASPIRE II. Lifestyle and risk factor management and use of drug therapies in coronary patients from 15 countries. Eur Heart $J$ 2001; 22: 554-572.

2. Recommendations by the Working Group on Cardiac Rehabilitation of the European Society of Cardiology. Long-term comprehensive care of cardiac patients. Eur Heart J 1992; 13 (suppl C): 1C-45C.

3. Rehabilitation after cardiovascular diseases, with special emphasis on developing countries: report of a WHO Committee. World Health Organ Tech Rep Ser 1993; 831: 1-122.

4. Wood D, De Backer G, Faergeman D, Graham I, Mancia G, Pyörälä K. Prevention of coronary heart disease in clinical practice. Recommendations of the Second Joint Task Force of European and other Societies on coronary prevention. Eur Heart J 1998; 19: 1434-1503. 
5. Ades PA. Cardiac rehabilitation and secondary prevention of coronary heart disease. $N$ Engl J Med 2001; 345: 892-902.

6. Pasquali SK, Alexander KP, Peterson ED. Cardiac rehabilitation in the elderly. Am Heart J 2001; 142 (5): 748-755.

7. Wenger NK, Froelicher ES, Smith LK, et al. Cardiac rehabilitation. Clinical practice guideline. No.17 Rockville, MD: U.S. Department of Health and Human Services, Public Health Service, Agency for Health Care Policy and Research and the National Heart, Lung, and Blood Institute. AHCPR No. 96-0672. October 1995.

8. Fletcher GF, Balady G, Blair SN, et al. Statement on exercise: benefits and recommendations for physical activity programs for all Americans. A statement for health professionals by the committee on exercise and cardiac rehabilitation of the council on clinical cardiology, American Heart Association. Circulation 1996; 94: 857-862.

9. Cobelli F, Tavazzi L. Relative role of ambulatory and residential rehabilitation. Journal of Cardiovascular Risk 1996; 3: 172-175.

10. Monpere C. Cardiac Rehabilitation: Guidelines and Recommendations. Dis Manage Health Outcomes 1998; 4: 143-156.

11. Giannuzzi P, Saner H, Bjornstad H, Fioretti P, Mendes M, Cohen-Solal A, Dugmore L, Hambrecht R, Hellemans I, McGee H, Perk J, Vanhees L, Veress G, Working Group on Cardiac Rehabilitation and Exercise Physiology of the European Society of Cardiology. Secondary prevention through cardiac rehabilitation: position paper of the Working Group on Cardiac Rehabilitation and Exercise Physiology of the European Society of Cardiology. Eur Heart $J$ 2003; 24 (13): 1273-8.

12. Balady GJ, Ades PA, Comoss P, Limacher M, Pina IL, Southard D, Williams MA, Bazzarre T. Core components of cardiac rehabilitation/secondary prevention programs. A statement for healthcare professionals from the American Heart Association and the American Association of Cardiovascular and Pulmonary Rehabilitation. Circulation 2000; 102: 1069-1073.

13. Smith SC, Blair SN, Bonow R, Brass L, Cerqueira MD, Dracup K, Fuster V, Gotto A, Grundy SM, Miller NH, Jacobs A, Jones D, Krauss RM, Mosca L, Octene, Pasternak RC, Pearson T, Pfeffer MA, Starke RD, Taubert KA AHA/ACC guidelines for preventing heart attack and death in patients with atherosclerotic cardiovascular disease: 2001 update. Circulation 2001; 104: 1577-1579.

14. Fletcher GF, Balady GJ, Ezra A, et al. AHA scientific statement: exercise standards for testing and training. Circulation 2001; 104: 1694-1740.
15. Taylor RS, et al. Exercise-based rehabilitation for coronary heart disease. The Cochrane Review Am J Med 2004; 116: 682-697.

16. Dugmore LD, Tipson RJ, Phillips MH, Flint EJ, Stentiford NH, Bone MF, Littler WA. Changes in cardiorespiratory fitness, phychological wellbeing, quality of life, and vocational status following a 12 month cardiac exercise rehabilitation programme. Heart 1999; 81: 359-366.

17. Oldridge NB, Guyatt GH, Fischer ME, Rimm AA. Cardiac rehabilitation after myocardial infarction. Combined experience of randomized clinical trials. JAMA 1988; 260: $945-950$

18. O'Connor GT, Buring JE, Yusufr S, Goldhaber SZ, Olmstead EM, Paffenbarger RS, Hennekens CH. An overview of randomized trials of rehabilitation with exercise after myocardial infarction. Circulation 1989; 80: 234-244.

19. Vanhees L, McGee HM, Dugmore LD, Schepers D, van Daele P on behalf of the Carinex Working Group. A representative study of cardiac rehabilitation activities in European Union Member States: the Carinex Survey. $J$ Cardiopul Rehabil 2002 (in press).

20. Hambrecht R, Gielen S, Linke A, Fiehn E, Yu J, Walther C., Schoene N, Schuler G. Effects of exercise training on left ventricular function and peripheral resistance in patients with chronic heart failure: A randomized trial. JAMA 2000; 283: 3095-3101.

21. Belardinelli R, Georgiou D, Cianci G, Purcaro A. Randomized controlled trial of long-term moderate exercise training in chronic heart failure: effects on functional capacity, quality of life, and clinical outcome. Circulation 1999; 99: 1173-1182.

22. Giannuzzi P, Tavazzi L, Meyer K, Perk J, Drexler H, Dubach P, Myers J, Opasich C, Meyers J, for the Working Group on Cardiac Rehabilitation \& Exercise physiology and Working Group on Heart Failure of the European Society of Cardiology. Recommendations for exercise training in chronic heart failure patients. Eur Heart J 2001; 22: $125-135$.

23. Urbinati S, Fattirolli F, Tramarin R, Chieffo C, Temporelli P, Griffo R, Belardinelli R, Vaghi P, Briolotti L; Gruppo Italiano di Cardiologia Riabilitativa e Preventiva. The ISYde Project. A survey on Cardiaca Rehabilitation in Italy. Monaldi Arch Chest Dis 2003; 60 (1): 16-24.

24. Giannuzzi P. Cardiologic rehabilitation from the hospital to medical practice. Monaldi Arch Chest Dis 2003; 60 (3): 241-5.

25. Cardiologia Riabilitativa e Prevenzione Secondaria delle Malattie Cardiovascolari. Linee guida nazionali di riferimento 9 ASSR; Piano Nazionale Linee Guida, 2005. 